\title{
Nanoparticle destruction by X-ray absorption
}

\author{
J. B. A. Mitchell ${ }^{1}$, C. Rebrion-Rowe ${ }^{1}$, J.-L. LeGarrec ${ }^{1}$, G. Taupier ${ }^{1}$, N. Huby ${ }^{1}$, and M. Wulff ${ }^{2}$ \\ 1 Equipe dAstrochimie Expérimentale, P.A.L.M.S., UMR 6627 du CNRS, Université de Rennes I, \\ 35042 Rennes Cedex, France \\ 2 European Synchrotron Radiation Facility (ESRF), BP 220, 38043 Grenoble cedex, France
}

Received 9 November 2001 / Accepted 18 February 2002

\begin{abstract}
X-ray absorption by nanometer sized soot particles in an ethylene flame has been studied using a beam from the ESRF synchrotron. This absorption appears to lead to the destruction of the particles. Application of this phenomenon to the release of molecules from the surface of interstellar dust grains is discussed.
\end{abstract}

Key words. methods: laboratory - ISM: dust, extinction - X-rays: ISM

\section{Introduction}

Interstellar dust particles can act as active surfaces for the catalytic formation of molecular species. The very cold $(10 \mathrm{~K})$ interstellar dust particles can also act as condensation surfaces onto which interstellar molecules will deposit and thus be depleted from the gas phase. A question that remains poorly answered is how molecules once formed or deposited could be released from the surface of the cold dust grains and thus appear as free molecular species that are detected by astronomical observations. A number of workers have performed experimental and theoretical studies of molecule formation and desorption from surfaces (Watson \& Salpeter 1972; Léger et al. 1985; Duley 1996) but many uncertainties still remain concerning such processes. One hypothesis is that the energy released by exothermic chemical reactions on the surface of the particles can serve to project the molecules so formed out into space (Hollenbach \& Salpeter 1970; Vittadini \& Selloni 1995). Since the dust particles are often bombarded by $\mathrm{X}$-rays and cosmic ray particles, capable of penetrating the vast interstellar clouds, it is also natural to assume that there may be processes whereby this energy can be absorbed by the dust and result in molecular desorption. Such processes involve the heating of the dust particles leading to evaporation (Aanestad et al. 1979; Gauger et al. 1990; Voit 1991) or even the triggering of explosive reactions involving reactive radicals that eject condensed species (d'Hendecourt et al. 1982; Shalabiea \& Greenberg 1994). The photoemission of electrons from irradiated dust particles has also been examined by astrophysicists

Send offprint requests to: J. B. A. Mitchell, e-mail: mitchell@univ-rennes1.fr
(Watson 1972; Verstraete et al. 1990; Dwek \& Smith 1996) as it may represent a heating mechanism for interstellar clouds. A recent synchrotron radiation experiment bears direct relevance to this problem and may have identified a new mechanism for the release of molecular species from grain surfaces into the interstellar medium.

\section{Experimental results}

The basic idea behind the experiment was to pass an Xray beam from a synchrotron through a cylindrically symmetric ethylene diffusion flame and to detect any ionisation produced by X-ray absorption by soot particles, using an electrically biased wire probe, located just above where the beam passed through the flame. Ethylene diffusion flames have been very well characterised and it is known that typical soot particle densities within particlerich zones in such flames are of the order of $10^{11} \mathrm{~cm}^{-3}$ and that their diameters range from 10-200 nm (Santoro et al. 1983). They represent therefore a very inexpensive system for the production and study of nano-particles. The structure of soot particles can be compared with that of carbonacious grains found in the interstellar medium (Mathis \& Whiffen 1988; Dwek 1997; Vaidya et al. 2001). Soot particles, sampled from hydrocarbon flames, have been subjected to a wide range of chemical and physical analytical techniques including Rayleigh scattering and visible light depolarisation measurements (Santoro et al. 1983) to determine particle density, size and gross structure, and scanning (Saito et al. 1991) and transmission (Vander Wal 1997; Ishiguro 1997) electron microscopy for fine details of the structure. Laser microprobe mass spectrometry (Dobbins et al. 1996), laser desorption mass spectrometry (Majidi et al. 1999) and real time mass 


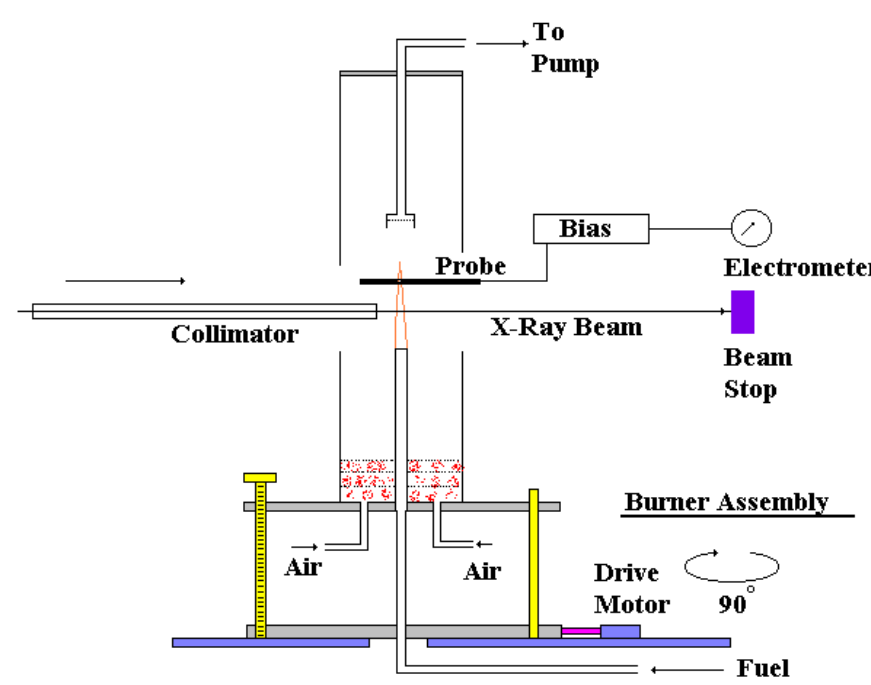

Fig. 1. Schematic of the X-ray absorption experiment showing the burner assembly that can be moved horizontally and vertically so that the soot density in the flame can be mapped. The electrically biased probe is used to collect the ionisation products formed naturally in the flame and due to ionisation of soot particles and background air molecules by X-ray absorption.

spectrometry (Reilly et al. 2000) have been used for chemical analysis of the particles. Young soot particles take the form of spherical particles with diameters of a few $\mathrm{nm}$ and contain a range of aromatic and polyaromatic hydrocarbon compounds. As the particles age in the flame, they lose hydrogen and the material becomes more graphite-like. The particles agglomerate into fractal-like forms (Filippov et al. 2000) consisting of small spherical particles connected by branches, these structures having dimensions from tens of nanometres up to microns in size. It is also possible (Mitchell \& Miller 1989) to dope soot particles by introducing metallic additives into the fuel and so one can produce soot particles that contain, for example, iron oxide in their cores. One can also create silicon dioxide and titanium dioxide nanoparticles in flames and in fact that is a major industrial technique for the manufacture of such species (Pratsinis 1998; Wooldridge 1998). The present article will, however, discuss experiment performed on natural soot particles. The experiment (illustrated schematically in Fig. 1) was carried out using white light on the ID09 undulator beamline at the European Synchrotron Radiation Facility (ESRF) in Grenoble, France. The photon flux was $3 \times 10^{11}$ photons $/ \mathrm{s}$ and the beam dimensions at the flame were $50 \mu \mathrm{m} \times 50 \mu \mathrm{m}$. The X-rays in the beam ranged in energy from 10 to 30 $\mathrm{keV}$. The flame was formed on a $11 \mathrm{~mm}$ diameter cylindrical tube through which the fuel flowed. This tube was surrounded by a $100 \mathrm{~mm}$ diameter tube through which air from a compressor was made to pass. A fuel flow rate of $62 \mathrm{ml} / \mathrm{min}$ of ethylene and $39 \mathrm{l} / \mathrm{min}$ of air yielded a flame with a visible height of $40 \mathrm{~mm}$. The flame was scanned horizontally and vertically by the X-ray beam in order to map out the location of soot particles formed during the combustion process. They were detected by measuring the

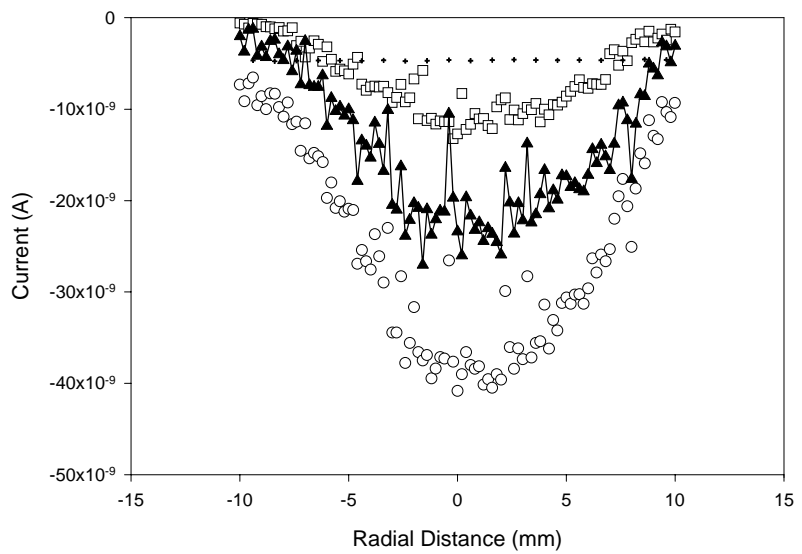

Fig. 2. Measured ionisation current to a positive probe at height of $32 \mathrm{~mm}$ above the burner. The crosses show current due to X-ray ionisation of background air without flame present. Open triangles and open squares are ionisation current with and without the X-ray beam respectively. The solid circles represent the difference in these two measurements with the contribution due to air absorption subtracted.

ionisation current, resulting from X-ray absorption, using a $1 \mathrm{~mm}$ diameter, stainless steel rod, to which a $\pm 20 \mathrm{~V}$ electrical bias was applied using a floating power supply that was connected to an electrometer. Prior to performing measurements on the actual flame, ionisation produced by passage through the background air and while ethylene gas flowed through the burner were performed in order to determine the baseline ionisation signal. It was found that air and ethylene produced similar ionisation yields of about $-5 \times 10^{-9} A$ as measured with a positively biased probe. Figure 2 shows the negative ionisation current measured across the flame when the X-ray beam intersected the flame at a height of $32 \mathrm{~mm}$ above the burner throat. It can be seen that the X-ray induced signal is about a factor of five larger than the background gas ionisation rate. Absorption of high energy X-rays in molecules and solids is in fact atomic-like for energies greater than $50 \mathrm{eV}$ (Henke et al. 1982). This means that one can take the cross section for absorption by individual elemental atoms and sum over the number of such atoms in the target. For $20 \mathrm{keV}$ X-rays, the corresponding absorption cross sections for carbon, nitrogen and oxygen are $4.1 \times 10^{-24} \mathrm{~cm}^{2}, 8.27 \times 10^{-24} \mathrm{~cm}^{2}$ and $1.57 \times 10^{-23} \mathrm{~cm}^{2}$ respectively. For air therefore, one can say that the absorption cross section is:

$$
\begin{array}{r}
\sigma(\text { air })=2 \times\left\{0.8 \times 8.27 \times 10^{-24}+\right. \\
\left.=.2 \times 1.57 \times 10^{-23}\right\} \\
=1.95 \times 10^{-23} \mathrm{~cm}^{2} .
\end{array}
$$

At room temperature and atmospheric pressure, the density of molecules is $2.4 \times 10^{19} \mathrm{~cm}^{-3}$ and the distance over which the ionisation signal was collected was approximately $5 \mathrm{~cm}$. Using the Beer-Lambert absorption law:

$$
I=I_{0} \exp (n l \sigma)
$$

where $I_{0}$ is the incident beam intensity, $I$ is the beam intensity after a distance $l$, the absorption length, $n$, 


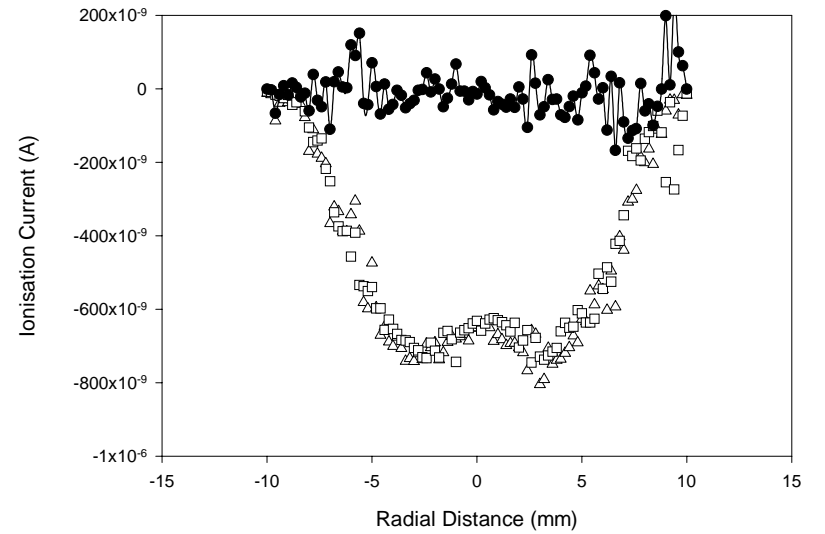

Fig. 3. Measured ionisation current to a positive probe at height of $2 \mathrm{~mm}$ above the burner. Open triangles and open squares are ionisation current with and without the X-ray beam respectively. The solid circles represent the difference in these two measurements with the contribution due to air absorption subtracted.

the number density of target molecules, and $\sigma$, the absorption cross section, one finds a ratio of the number of absorption events to the incident beam intensity: $\left(I_{0}-I\right) / I_{0}=2.4 \times 10^{-3}$ for absorption in the background air. Measurements of optical absorption by soot particles in ethylene flames (Santoro et al. 1983) show that the volume of the flame occupied by soot particles, (the soot volume fraction $f_{\mathrm{v}}$ ) is of the order of $10^{-5}$ in regions where the soot concentration is highest. Taking the density of soot particles to be similar to graphite $\left(r=2.2 \mathrm{~g} \mathrm{~cm}^{-3}\right)$ and using the Avogadro number $\left(6 \times 10^{23}\right)$, one finds that the number density of carbon atoms in the flame is $1.1 \times 10^{18} \mathrm{~cm}^{-3}$. The path length of the beam through the flame is roughly equivalent to the diameter of the burner $(1.1 \mathrm{~cm})$ and so again using the Beer-Lambert law one obtains a value of $\left(I_{0}-I\right) / I_{0}=4.5 \times 10^{-6}$, i.e. a factor of 532 times less than the number of absorption events occurring in the background air. Given the large X-ray induced signal seen it would appear that absorption by the soot particles results in an intense release of electrons. Figure 3 shows a similar plot but taken $2 \mathrm{~mm}$ above the burner throat. In this case there is essentially no signal generated due to X-ray ionisation of soot particles and this is to be expected since other studies (Santoro et al. 1983) have shown that soot particle density is low in this zone. Similar plots were obtained for the collection of positive species though the measured currents were smaller over most of the flame height. The current measured by a probe in an atmospheric flame (Fialkov 1997) is proportional to the fourth power of the particle mobility, $(\mu)^{1 / 4}$. Thus highly mobile free electrons, released from soot particles will yield larger measured currents that the heavy positive species left behind. It was found that the electron current peaked at around $20 \mathrm{~mm}$ above the burner and then declined between 20 and $35 \mathrm{~mm}$, eventually reaching a value similar to that of the positive current, (that had been constantly rising over the same height range). The reason for this decline in the measured negative current is that free electrons attach to neutral heavy soot particles, whose number density rises with height-above-burner and thus the mobility of the negative charge carriers drops sharply. The equality in the positive and negative currents indicates that at this stage of the combustion, the electrical charge is carried by solid particles rather than by free electrons and ions.

\section{Discussion}

High energy X-ray photons are absorbed primarily by atomic inner-shell electrons (rather than outer-shell valence electrons as is the case for ultra-violet absorption). The electrons are ejected from the atom and thus a primary ionisation event occurs that leaves an inner-shell vacancy in the target atom. This vacancy is then filled by an electron from a higher level either of the target atom or of a neighbouring atom. In this event a high energy photon can be emitted (fluorescence) or the energy release can lead to the ejection of another electron from the atom (the Auger effect). If a lower lying electron is ejected then a second such Auger process can occur etc. This is known as an Auger cascade though in fact for carbon only one $262.4 \mathrm{eV}$ Auger electron is released (Dwek \& Smith 1996). Since the target atom is located within a solid matrix, the departing primary photoelectron and the Auger electron must traverse this material in order to escape. One can determine the distance needed to stop an electron in the material using the range equation (Voit 1991):

$$
\begin{aligned}
\log (R)= & -8.1070+1.0596 \log \left(E_{\mathrm{el}}\right)-0.27838 \log ^{2}\left(E_{\mathrm{el}}\right) \\
& +0.11741 \log ^{3}\left(E_{\mathrm{el}}\right)-0.010731 \log ^{4}\left(E_{\mathrm{el}}\right)
\end{aligned}
$$

where $R$ is the range of electrons in matter expressed in units of $\mathrm{g} / \mathrm{cm}^{2}$ and $E_{\mathrm{el}}$ is the electron energy in $\mathrm{eV}$. The mean free path can be determined by dividing this quantity by the material density $\rho$. Thus the mean free path of a $20 \mathrm{keV}$ electron in carbon is of the order of $4000 \mathrm{~nm}$ so it will most likely simply escape the particle without further interaction. That for a $260 \mathrm{eV}$ electron is of the order of $5 \mathrm{~nm}$ however, and so it will undergo inelastic collisions with other atoms in the solid, resulting in secondary electron emission. Thus the primary X-ray absorption can lead to the ejection of a number of electrons. If such a process occurs in an electrically unbiased bulk solid, the electrons will probably return to the surface thus reneutralising it. In the case of a small particle, it is quite likely that the electrons will escape if the size of the particles is small compared to the electron mean free path in the surrounding gas and this will leave the particle with a net positive charge. (Natural soot particles usually are electrically charged, whether positively due to thermionic emission or negatively due to electron or negative ion attachment. Flames also contain ions formed via chemical processes (Fialkov 1997). These phenomena are responsible for the current measured with the flame but without the X-ray beam). The positive charge built up on the particle will give rise to an electric potential. For a spherical particle the value of this potential is given by:

$\phi=e z /\left(4 \pi \epsilon_{0} a\right)$ 
where $e$ is the electronic charge, $\epsilon_{0}$ the permittivity of free space and $a$ is the particle radius. $z$ is the number of charges on the particle. For a $10 \mathrm{~nm}$ diameter particle therefore:

$\phi=0.288 z$ Volts.

This is a small potential but given the small size of the particle, the resulting electric field

$(E=\phi / a)$

generated can be enormous. Thus for example, if a $10 \mathrm{~nm}$ diameter particle loses 10 electrons, the potential generated is 2.88 volts and the resulting electric field around it is $5.76 \times 10^{8} \mathrm{~V} / \mathrm{m}$ ! Studies (Rinzler et al. 1995; Ferrari et al. 1999; Bonard et al. 1999) have shown that fields of the order of $10^{6}-10^{7} \mathrm{~V} / \mathrm{m}$ induce field emission of electrons from carbon nanotubes. This process of primary, secondary and tertiary electron emission is illustrated schematically in Fig. 4.

Electron microscopic and mass spectrometric studies (Saito 1991; Ball \& Howard 1971; Dobbins et al. 1996; Filippov et al. 2000) of soot particles have shown that they display a fractal-like, agregated structure and at medium heights in the flame they consist of both graphite like and aromatic or polyaromatic materials. It is very likely therefore that if strong electric fields can be induced within such agregated particles by x-ray absorption as discussed above, that this would lead not only to runaway electron and positive ion emission but also to subsequent prompt disruption of the aggregate (Draine \& Salpeter 1979; Chang et al. 1987; Ball \& Howard 1971; Fruchter et al. 2001). Indeed a series of experiments has been performed by Grün and co-workers (Svestka \& Grün 1992; Cermak et al. 1995) in which aggregate particles have been stored in an radiofrequency quadrupole trap and bombarded by ion or electron beams, and these experiments have shown that such fragmentation does occur. Field emission of electrons and ions has also been observed in these experiments.

In order for fragmentation to occur, the electrostatic stress $S_{\mathrm{e}}$ induced in the particles due to charging must exceed the tensile strength $S_{\mathrm{t}}$ of the material. (Most of the discussions in the astrophysical literature have used c.g.s units for such comparisons and so we shall also). Known values of tensile strength for relevent materials are $5 \times 10^{8}$ dyne $\mathrm{cm}^{-2}$ for graphite, $10^{6}-10^{8}$ dyne $\mathrm{cm}^{-2}$ for silicates 1992. Pinter et al. (1989) have examined the tensile strength of a synthesized fluffy aggregate material consisting of spherical glass cores $(<30 \mu$ m diameter $)$ with a hydrocarbon (N-pentacosane) mantle and measured a tensile strength ranging from $10^{5}-10^{7} \mathrm{~Pa}\left(10^{6}-10^{8}\right.$ dyne $\left.\mathrm{cm}^{-2}\right)$. The electrostatic stress is given by:

$S_{\mathrm{e}}=(1 / 4 \pi)(U / a)^{2}$

where $U$ is the electrostatic potential measured in esu $\left(1 \mathrm{~V}=1 / 2.9979 \times 10^{2} \mathrm{esu}\right)$. Thus for the $2.88 \mathrm{~V}$ potential generated by the loss of 10 electrons from a $10 \mathrm{~nm}$ diameter particle, this will produce an electrostatic stress $S_{\mathrm{e}}$

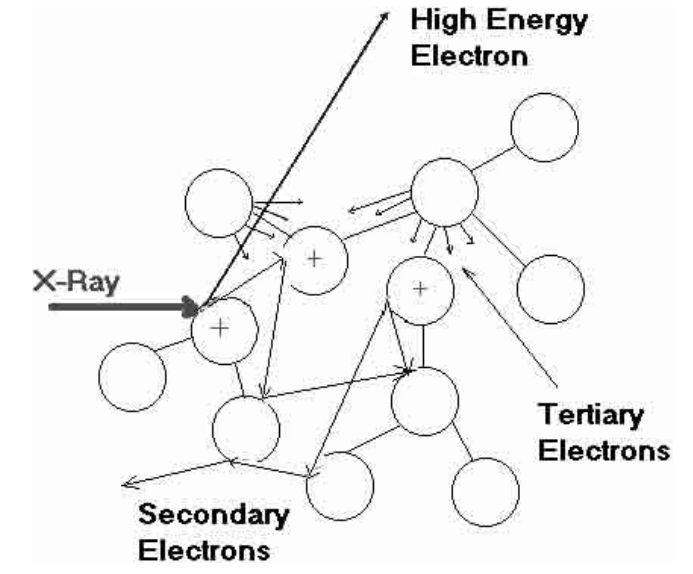

Fig. 4. Schematic of the proposed electron emission process. The absorption of the incoming X-ray yields a primary high energy electron that leaves the particles without further reaction. An Auger electron is also released that makes further collisions with the aggregate yielding secondary electrons some of which also leave the particle. This results in a buildup of positive charge and subsequent high electric fields between the primary particles. These fields induce field emission of tertiary electrons causing further positive charge buildup and eventual Coulomb induced fragmentation of the aggregate.

of $7.3 \times 10^{6}$ dyne $\mathrm{cm}^{-2}$. Hence this is sufficient to cause fragmentation of a fluffy grain.

Some fraction of interstellar dust particles are believed to be carbonaceous in form (Mathis et al. 1977; Mathis \& Whiffen 1988) and to have irregular structures. In a recent article, Dominik \& Tielens (1997) have examined how such structures can be formed. As noted above, such particles are often subject to X-ray irradiation and therefore similar ionisation processes can be expected to occur with these particles as have been seen in the synchrotron radiation experiment discussed here. If these particles are coated with molecular species, either formed in-situ or condensed from the gas phase, then such disruptive phenomena will be very effective in releasing these molecules back into space. We believe that this is the first time that actual experimental evidence for such an X-ray induced disruptive process in free nano-particles has been reported. It should be mentioned that a recent article by Najita et al. (2001) has discussed the thermal release of molecules from the surface of fluffy aggregated grains. This is caused by the spot heating of poorly thermally connected aggregated structures. Again this would be an example of a phenomenon that relies upon the aggregate nature of the particle.

There is other experimental information that shows that small particles can give rise to anomalous electron emission effects. In a series of experiments, Schmidt-Ott and co-workers (Schmidt-Ott et al. 1980; Burtscher et al. 1984, 1985; Müller et al. 1988a) observed a large enhancement of photoemission yields from $5 \mathrm{~nm}$ diameter silver particles, irradiated by $10 \mathrm{eV}$ photons. This enhancement could not be explained by photoemission theory (Müller et al. 1988b). Anomalous electron emission has also been 
seen when magnesium is burned in air (Markstein 1967; Mitchell \& Miller 1989) and when granular deposits of magnesium oxide are irradiated by ultra-violet light (Feist 1968). It seems reasonable to suppose that these observations can also be interpreted in terms of a runaway field emission phenomenon though in none of these experiments was the resulting state of the emitting particle studied.

A new apparatus is under construction that will address this point. Experiments are planned where time-offlight mass spectrometry will be used to determine the masses of the positive particles produced during the absorption process. It is also planned to study the process as a function of incident photon energy, extending the measurements into the vacuum ultra-violet (VUV) region of the spectrum.

Acknowledgements. The financial support of the European Office of Aerospace Research and Development (EOARD), Air Force Office of Scientific Research (AFOSR) and of the European Synchrotron Radiation Facility, (ESRF) are gratefully acknowledged. Thanks are also due to the European Synchrotron Radiation Facility for granting us the beamtime necessary to perform the experiment and to Daniel Travers and René Jaffré of the University of Rennes for the construction of the apparatus. Useful conversations with Eli Dwek, Bruce Draine and Eberhard Grün are also acknowledged.

\section{References}

Aannestad, P. A., \& Kenyon, S. J. 1979, ApJ, 230, 771

Ball, R. T., \& Howard, J. B. 1971, 13th (Internation) Symposium on Combustion, The Combustion Institute, Pittsburgh, 353

Bonard, J. M., Salvetat, J. P., Stockli, T., Forro, L., \& Chatelain, A. 1999, Appl. Phys. A, 69, 245

Burtscher, H., Schmidt-Ott, A., \& Siegmann, H. C. 1984, Z. Phys. B, 56, 197

Burtscher, H., \& Schmidt-Ott, A. 1985, Surf. Sci., 156, 735

Cermak, I., Grün, \& Svestka, J. 1995, Adv. Space Res., 10, 59

Chang, C. A., Schiano, A. V. R., \& Wolfe, A. M. 1987, ApJ, 322,180

d'Hendecourt, L. B., Allamandola, L. J., Baas, F., \& Greenberg, J. M. 1982, A\&A, 109, L12

Draine, B. T., \& Salpeter, E. E. 1979, ApJ, 231, 77

Dobbins, R. A., Govatzidakis, G. J., Lu, W., Schwartzmann, A. F., \& Fletcher, R. A. W. 1996, Combust. Sci. Tech., 121, 103

Dominik, C., \& Tielens, A. G. G. M. 1997, ApJ, 480, 647

Duley, W. W. 1996, ApJ, 471, L57

Dwek, E., \& Smith, R. K. 1996, ApJ, 459, 686

Dwek, E. 1997, ApJ, 484, 779

Feist, W. M. 1968, Advances Electronics Electron Physics Supplement 4, ed. L. Marton, \& A. B. El-Karch (Academic Press, NY)
Ferrari, A. C., Satyanarayana, B. S., Robertson, J., et al. 1999 Europhy. Lett. 46, 245

Fialkov, A. B. 1997, Prog. Ener. Combust. Sci., 23, 399

Filippov, A. V., Zurita, M., \& Rosner, D. E. 2000, J. Colloid. Interface Sci., 229, 261

Fruchter, A, Krolik, J. H., \& Rhoads, J. E. 2001, ApJ, 563, 597

Gauger, A., Sedlmayr, E., \& Gail, H. P. 1990, A\&A, 235, 345

Svestka, J., \& Grün, E. 1992, Hypervelocity Impacts in Space, ed. J. A. M. McDonell (Univ. of Kent Press, Canterbury), 139

Henke, B. L., Lee, P., Tanaka, T. J., Shimabukuro, R. L., \& B. K., Fajikawa, B. K. 1982, At. Data Nucl. Data Tables, 27,1

Hollenbach, D., \& Salpeter, E. E. 1970, J. Chem. Phys., 53, 79

Ishiguro, T., Takatori, T., \& K., Akihama, K. 1997, Combust. Flame, 108, 231

Léger, A., Jura, M., \& Omont, A. 1985, A\&A, 144, 147

Majidi, V., Saito, K., Gordon, A. S., \& Williams, F. A. 1999, Combust. Sci. Tech., 145, 37

Markstein, G. H. 1967, 11th Int. Symp. Combust. (The Combustion Institute, Pittsburgh), 219

Mathis, J. S., \& Whiffen, G. 1988, ApJ, 341, 808

Mathis, J. S., Rumpl, W., \& Nordsieck, K. H. 1977, ApJ, 217, 425

Mitchell, J. B. A., \& Miller, D. J. M. 1989, Combust. Flame, 75,45

Müller, U., Schmidt-Ott, A., \& Burtscher, H. 1988a, Z. Phys. B, 73,103

Müller, U., Burtscher, H., \& Schmidt-Ott, A. 1988b, Phys. Rev. B, 38, 7814

Najita, J., Bergin, E. A., \& Ullom, J. N. 2001, ApJ, 561, 880

Pinter, S., Blum, J., \& Grün, E. 1989, Proc. Int. Wkshp. Phys. Mech. Cometary Materials, Múnster, Germany (ESA SP-302 Dec.1989), 215

Pratsinis, S. E. 1998, Combust. Sci. Tech., 24, 197

Reilly, P. T. A., Gieray, R. A.,Whitten, W. B., \& Ramsey, J. M. 2000, Combust. Flame, 122, 90

Rinzler, A. G., Hafner, J. H., Nikolaev, P., et al. 1995, Science, 269,1550

Saito, K., Gordon, A. S., Williams, F. A., \& Streibel, T. 1991, Combust. Sci. Tech., 80, 103

Santoro, R. J., Semerjian, H. G., \& Dobbins, R. A. 1983, Combust. Flame, 51, 203

Schmidt-Ott, A., Schurtenberger, P., \& Siegmann, H. C. 1980, Phys. Rev. Lett., 45, 1284

Shalabiea, O. M., \& Greenberg, J. M. 1994, A\&A, 290, 266

Vaidya, D. B, Gupta, R., Dobbie, J. S., \& Chylek, P. 2001, A\&A, 375, 584

Vander Wal, R. 1997, Combust. Sci. Tech., 126, 333

Verstraete, L., Léger, A., d'Hendecourt, L. Dutuit, O., \& Défourneau, D. 1990, A\&A, 37, 436

Vittadini, A., \& Selloni, A. 1995, Chem. Phys. Lett., 235, 334

Voit, G. M. 1991, ApJ, 379, 122

Wooldridge, M. S. 1998, Combust. Sci. Tech., 24, 63

Watson, W. D., \& Salpeter, E. E. 1972, ApJ, 174, 321

Watson, W. D. 1972, ApJ, 176, 103 